\title{
External defibrillation on an implantable defibrillator
}

\author{
John Chiladakis $\cdot$ Fani Zagkli \\ Dimitrios Alexopoulos
}

Received: 13 August 2013/ Accepted: 3 September 2013/Published online: 6 October 2013

(C) Japanese Society of Anesthesiologists 2013

Keywords External defibrillation - Implantable defibrillator

To the Editor:

Despite the best efforts of healthcare providers, guideline-conform treatment failure is expected to occur, particularly in real-world emergency situations [1]. In the case reported here, a 79-year-old man with a history of coronary artery disease and implantation of a cardioverter defibrillator (ICD) presented to the emergency room with recurrent ventricular tachycardia. The junior physician-incharge applied an external biphasic DC shock of $200 \mathrm{~J}$ to the patient, holding the paddle in one hand and placing it directly over the ICD (Electronic Supplementary Material Fig. 1)—but without success. Arrhythmic stability was finally achieved by multiple, appropriate ICD shocks and intravenous amiodarone. Subsequent device interrogation and defibrillation testing of the ICD showed no instance of device malfunction. Coronary angiography revealed arteries free of significant stenoses.

Strong electromagnetic interference induced by transthoracic DC shocks may cause permanent damage or alter the operation of the implanted device [2]. When attempting external defibrillation, clinicians are advised to place the paddles/pads as far as possible from any pulse generator

Electronic supplementary material The online version of this article (doi:10.1007/s00540-013-1710-9) contains supplementary material, which is available to authorized users.

J. Chiladakis $(\bowtie) \cdot$ F. Zagkli $\cdot$ D. Alexopoulos

Cardiology Department, Patras University Hospital,

26500 Rion, Patras, Greece

e-mail: chil@otenet.gr and to choose biphasic over monophasic shock waveforms. A less deleterious effect might be expected if the orientation of the applied electric field is perpendicular-and not parallel - to the device with its lead(s), whereas if the device is located in the left pectoral region, an anteriorapex paddle position may be also acceptable. With an anterior-posterior electrode orientation and a distance between device and hand-held shock electrode of $>8 \mathrm{~cm}$, Manegold et al. [3] did not observe any dysfunction in patients with right- or left-sided implanted pacemakers or ICDs treated with external cardioversion for atrial fibrillation.

Conflict of interest None.

\section{References}

1. Grzeskowiak M, Plotek W, Podlewski R. The quality of defibrillation performance among students of the University of Medical Sciences. J Anesth. 2011;25:627-9.

2. [No authors listed]. Part 6: advanced cardiovascular life support. Section 2: defibrillation. European Resuscitation Council. Resuscitation. 2000;46:109-13.

3. Manegold JC, Israel CW, Ehrlich JR, Duray G, Pajitnev D, Wegener FT, Hohnloser SH. External cardioversion of atrial fibrillation in patients with implanted pacemaker or cardioverterdefibrillator systems: a randomized comparison of monophasic and biphasic shock energy application. Eur Heart J. 2007;28:1731-8. 